\title{
Sciendo
}

Transport and Telecommunication, 2022, volume 23, no. 1, 11-24

Transport and Telecommunication Institute, Lomonosova 1, Riga, LV-1019, Latvia

DOI $10.2478 / t t j-2022-0002$

\section{CRASH DISTRIBUTION DATASET: DEVELOPMENT AND VALIDATION FOR THE UNDIVIDED RURAL ROADS IN OROMIA, ETHIOPIA}

\author{
Alamirew Mulugeta Tola ${ }^{1,2}$, Tamene Adugna Demissie ${ }^{2}$, Fokke Saathoff ${ }^{1}$, \\ Alemayehu Gebissa ${ }^{1}$
}

\author{
${ }^{I}$ Faculty of Agricultural and Environmental Sciences, Chair of Geotechnics and Coastal Engineering \\ Rostock University \\ Justus-von-Liebig-Weg 6, LAGII, Rostock 18059, Germany \\ lolaal728@gmail.com; fokke.saathoff@uni-rostock.de; \\ alemayehu.Gebissa@uni-rostock.de \\ ${ }^{2}$ Faculty of Civil \& Environmental Engineering, Jimma Institute of Technology, Jimma University
} Jimma 378, Ethiopia

tamene.adugna@ju.edu.et

\begin{abstract}
Predicting the number of crashes that may occur as a result of specific highway features is critical in evaluating different treatment or design alternatives. Since different highway geometric characteristics can influence crash distribution datasets, Highway Safety Manual's (HSM's) predictive method encourages users to predict crashes based on their severity and collision type proportions. This study used crash data from rural two-way two-lane road segments in the Oromia region over seven years to develop Oromia's fixed crash distribution dataset on Interactive Highway Safety Design Model (IHSDM) software. The crash distribution dataset has two parts; the crash severity proportions and the collision type percentages. The developed Oromia's fixed crash distribution dataset was compared and validated against the default HSM crash configuration. As a result, the Crash Prediction Model (CPM) evaluation results confirmed that the developed crash severity proportion (the first part of the crash distribution dataset) estimates are more accurate and closer to the observed values. Furthermore, the findings show that crashes in the Oromia region are severer than in the states where the HSM crash configuration was developed. According to the second part of the crash distribution dataset evaluation (collision type percentage), the developed fixed crash distribution dataset outperforms the default HSM configuration in most collision type proportions, but not in all. For instance, from the ten collision type proportions developed, Right-Angle and sides-wipe collision proportions are predicted more precisely by the default HSM configuration. This points to the need for developing collision type proportion (the second part of the crash distribution dataset) as a function rather than a fixed configuration for a better result, based on the availability of complete crash data (i.e. crash location). In general, the study revealed that in order to exploit the full potential of HSM's predictive approach, researchers must develop a jurisdiction crash distribution dataset using local crash data. The methodology demonstrated in this study to develop the jurisdiction's crash distribution dataset has been validated as true thus, safety practitioners are encouraged to adopt it.
\end{abstract}

Keywords: crash distribution dataset, crash prediction model, HSM, IHSDM, Oromia, rural roads

\section{Introduction}

For highway engineers and road safety practitioners, reducing the occurrence of traffic crashes has continually been one of the significant duties worldwide. To achieve a significant reduction in road traffic crash fatalities and severe injuries, individual designers, transportation agencies, and concerned organizations should all work together to provide safe, efficient, and accountable road transportation. Predicting the number of crash frequencies that may occur due to a specific roadway design characteristic is essential for the evaluation of different alternatives in design and safety improvements. Hence, to be effective in highway safety improvements, safety engineers need to comprehend the associations between traffic crashes and roadway characteristics. Road safety studies have underlined the opportunities as well as safety challenges on highways (Burgess, 2005; Chandler \& Anderson, 2010; Tang et al., 2018; Tola \& Gebissa, 2019). In Ethiopia, most of the techniques of road safety analysis used by transport authorities and safety experts are the traditional descriptive approaches that are dedicated to quantify and summarize crash information.

The recent advancement in road safety research from descriptive techniques to more radical and statistically validated methods of predictive and quantitative approaches has piqued the interest of road safety practitioners. In the field of road safety, examining the safety outcomes for specific treatments is 
critical in improving highway safety. Current studies and practices have validated that highway planning and design that possess a scientific, consistent, and proactive approach can add a significant safety improvement. The American Association of State Highway and Transportation Officials (AASHTO) had published the Highway Safety Manual (HSM) to introduce a science-based predictive approach for safety practitioners and transport authorities ((AASHTO), 2010). The HSM directs statistical and scientific analysis techniques to quantify the safety outcomes of alternatives and mitigations in design, operation, and maintenance through the development of crash modification factors (CMFs). Part $\mathrm{C}$ of the Highway Safety Manual presents CPM approaches to quantify the safety associations with different facility types. Factors such as roadway characteristics, traffic volume and characteristics, and weather conditions are features that influence the expected number of crashes in the predictive analysis. Driver behavior, in which its data are not always accessible also influences the expected number of crashes. The driving behavior of the specific local jurisdiction can be studied from crash data information but 'Driving simulator' experiments and 'Naturalistic driving' studies may provide an accurate deep understanding of the contribution of driving characteristics on crash occurrences (Ahmed \& Chalise, 2018).

For a limited fund and cost of engineering studies, road safety studies based on the expected safety performance function are a feasible way to identify potential roadway sites for improvement. The predictive models of HSM (see Equation 1) detect roadway sites with great promise for the reduction of crash frequency and severity. For the specified analysis period, the predictive method of HSM presents an organized technique to predict the expected average crash frequency by their respective crash distribution dataset (i.e. categorized by overall crashes, crash severity proportions, and collision type distribution). The predictive approach of HSM has four main features these are; Safety Performance Function (SPF), Crash Modification Factor (CMF), Calibration Factor (C) and Crash distribution dataset ((AASHTO), 2010; Tarko et al., 2018). SPF is a statistical model that predicts the crash frequency of facilities based on the specified base conditions (HSMs specified base conditions). Whenever the characteristics of site conditions under the evaluation vary from the base condition, the corresponding CMFs are applied by multiplying it with the predicted crash frequency under base conditions. Calibration Factor (C) is also multiplied with SPF on behalf of differences in undetected crash frequency contributory factors such as weather, road user characteristics, vehicle characteristics, and crash reporting thresholds between states used to develop HSM and different countries and jurisdictions ((AASHTO), 2010). Thus, applying the HSM predictive method to different geographic locations without calibration may result in erratic crash predictions, which are being investigated by some researchers (B. Persaud et al., 2002; Sawalha \& Sayed, 2006). Whenever the observed crash frequency for the analysis years is available, the calibration factor can be obtained by dividing the observed crash frequency by that of the predicted one. Since HSM does not state any statistical techniques to check the validity of the calibration factor, safety practitioners should be careful during the application of the calibration procedure.

$$
N_{\text {Predicted }}=N_{S P F} \times C \times\left(C M F_{1} \times C M F_{2} \ldots \times C M F_{n}\right) .
$$

Where $N_{\text {predicted }}$ is the predicted average crash frequency of a site, $N_{S P F}$ is the safety performance function (SPF) for base conditions, $C$ is the calibration factor, and $C M F_{1}, C M F_{2} \ldots C M F_{n}$ are crash modification factors accounting for the variation in crash frequencies due to the safety treatments or site characteristics. Sites or locations with higher observed crashes than that of the predicted crash frequency needs additional investigations for the safety benefits.

It is a fact that driving behavior, roadway characteristics, the environment, weather conditions, and other situations in Ethiopia and the states used to develop HSM are quite different. Thus to have accurate crash estimates, the SPFs and crash distribution datasets of HSM should have to be calibrated to the local conditions of Ethiopia. The HSM calibration procedures are exhibited in Appendix A of the manual ((AASHTO), 2010).

\subsection{Application of HSM and IHSDM}

HSM allows local agencies and safety practitioners to develop and calibrate the individualized predictive components (i.e. SPF, CMFs, and crash distribution datasets). Many techniques can be used to customize the predictive HSM by combining its major elements, for instance, the study by Dixon et al., has investigated the possible options associated with calibration factors and crash distribution percentages under the HSMs default SPFs (Xie et al., 2011). Since the introduction of the Highway Safety Manual, highway safety engineers do not only use it as a standard in the predictive approach but also it is adopted by local and state transportation agencies in the United States and many other countries (Sacchi et al., 2012). For instance, HSM has been calibrated and implemented by the highway safety engineers, local and state transportation agencies, in the United States; Alabama, Florida, Louisiana, Missouri, Oregon, 
South Dakota, Texas, Utah, and Virginia (M. A. Abdel-Aty et al., 2014; Brimley et al., 2012; Fitzpatrick et al., 2006; Kweon et al., 2014; Mehta \& Lou, 2013; Qin et al., 2016; C. Sun et al., 2014; X. Sun et al., 2006; Xie et al., 2011) and in other countries such as Canada, Italy, and New Zealand (Koorey, 2010; Marchionna et al., 2012; Martinelli et al., 2009; B. Persaud et al., 2012). A study by Sacchi et al. (Sacchi et al., 2012) was made with a key objective of investigating the methodologies to assess the transferability of the overall HSM predictive algorithm and its components which are; the CMFs and baseline SPFs. The study was executed based on data conducted from rural two-lane undivided roads of Turin province in Italy. They compared the results of their studies with a similar study done in Canada as part of their study to amplify the conclusions on the transferability of the predictive HSM procedure in other countries (B. Persaud et al., 2012). The study result revealed the over-prediction of the HSM predictive model and suggesting the development of jurisdiction-specific SPFs and CMFs across Europe. The authors concluded the acceptability of using baseline SPFs and CMFs with local calibration factors by highlighting, the complexity of HSM transferability techniques and the need for substantial data and analysis.

Some of the studies have developed jurisdiction-specific Safety Performance Functions with locally available data in which it was found to give improved prediction estimates (Brimley et al., 2012; Kweon et al., 2014; Mehta \& Lou, 2013). In Kweon et al. (Kweon et al., 2014) study, the SPF variables and equations in the predictive HSM were modified for crash estimates using Virginia highways and crash data. Variables newer to HSM predictive approach such as; truck proportion and speed limit were included in the study executed to develop jurisdiction-specific SPFs (Brimley et al., 2012; Mehta \& Lou, 2013). In other studies, further inquiries have been discovered for instance; modeling lane width in nonlinear form rather than a linear form in the equation of SPFs shows improved estimates (Park \& AbdelAty, 2017) and the presence of an association between the shoulder and lane width as well as the necessity of including their correlation in SPFs model (Park \& Abdel-Aty, 2017).

In addition to the predictive HSM, SafetyAnalyst and Interactive Highway Safety Design Model (IHSDM) are the key road safety improvement tools for making efficient and effective design decisions (Qin et al., 2013). The crash prediction module of IHSDM, which is a faithful application of the predictive method of HSM in part C, has been used as a proactive road safety tool by providing the expected safety alternatives for the newly constructed highway (during the design phases) and existing roads ((FWHA), 2019). Nowadays, CPM of the Interactive Highway Safety Design Model (IHSDM) software, which is developed by the Federal Highway Administration, is the commonly used predictive approach. IHSDM software incorporates the four predictive components of HSM, which are (i) safety performance function (SPF), (ii) crash modification factors (CMFs), (iii) calibration factor (Cx), and (iv) crash distribution dataset (Tarko et al., 2018). Thus, the constraints in the calibration of the predictive HSM components can be carried out by the application of the IHSDM tool (Tarko et al., 2018). Many studies were made to assess the applicability of CPM in IHSDM software. For instance, Dominguez et al. (Dominguez-Lira et al., 2010) calibrated CPM in IHSDM with jurisdiction data on three highway sections in Spain. The results of their study showed that the IHSDM crash prediction with a calibrated dataset performed significantly better. They concluded that even if the application and interpretation of the CPM in IHSDM needs attention and expertise in the field, its application could be essential for the evaluation of existing highways and roadway rehabilitation projects.

Since different highway geometric characteristics may affect crash type and severity proportions, the predictive method of HSM encourages users to predict crashes by their respective proportions of severity and collision type. Predictive models by crash proportions yield a better estimate than crash frequency predictions with overall crash types (Jonsson et al., 2009). Crash prediction models based on specific crash distributions (i.e. by severity level and collision type) explores the associations between roadway geometric features and their accompanying crash types thus, helping policymakers, road safety engineers, and practitioners in making effective improvements on the severer crashes. In comparison to Rear-end collisions, Head-on collisions create more severe damages (Jonsson et al., 2009) and Run-offroad collisions are further associated with shoulder and lane widths than are Rear-end collisions (Zegeer et al., 1988). Roadway features significantly influence the number of crash types and their proportions. Persaud et al. employed an empirical Bayes before-after method by installing rumble strips on rural undivided highways (B. N. Persaud et al., 2004). Their study result discovered that the installation of rumble strips along the centerline has shown a reduction in all crash injuries and a significant reduction in opposite sideswipe and head-on crashes witnessed.

Because of the benefits of predicting average crash frequency in terms of crash severity and collision type proportion, the HSM predictive method provides default crash distribution datasets for rural two-way two-lane roadways ((AASHTO), 2010). HSM endorses the necessity of replacing this default crash distribution dataset with the fraction of local crash distribution estimates as part of the calibration procedure. Numerous studies found that the replacement of the fixed default crash proportions by the 
local crash history could improve the accuracy of crash prediction significantly (Brimley et al., 2012). In contrast, some studies argued that the assumption of fixed crash distribution datasets might be not true. For example, strong relationships between highway features and the possibilities of different crash severities and collision types have been explored by related studies (M. Abdel-Aty \& Keller, 2005; Haleem \& Abdel-Aty, 2010; Huang et al., 2008). In Qin et al. (Qin et al., 2019) study, they presumed that a crash severity proportion as a function rather than as a fixed proportion might predict crash frequency pretty well with higher accuracy. However, according to the study's result, crash severity proportion as a function does not improve the prediction accuracy. Thus, their study guarantees the effectiveness of a fixed crash proportion approach in the development of a jurisdiction-specific crash distribution dataset.

The current study has employed the HSM and Interactive Highway Safety Design Model (IHSDM) tool to compare and evaluate the prediction accuracy of crash distribution datasets of the developed Oromia's fixed crash distribution dataset and the default HSM crash dataset. "To exercise the full potential of IHSDM for a realistic and reliable estimate of crashes for local roads with certain geometric and traffic characteristics, the tool needs to be adjusted and calibrated" (Qin et al., 2013). An appropriate calibration is capable of inferring the difference between the jurisdictions' crash prediction models as well as local crash histories. Calibrating the crash prediction model of IHSDM is fundamentally the same as calibrating the predictive approach of HSM. The main objective of this study is to evaluate the effectiveness of developing a fixed jurisdiction crash distribution dataset using the local crash data for rural two-way two-lane roadway segments. To meet the study's objective, both the HSM's default values and the developed Oromia's-fixed crash distribution datasets had been compared for the trustfulness of crash predictions in terms of crash distribution. Thus, for the evaluation of the estimates by the developed local crash distribution datasets, the study presumes taking the default SPF models and CMFs (or with a calibration value of 1.0) of HSM in crash prediction analysis.

\section{Study area and data set}

\subsection{Study area description}

For many decades, the safety problems on rural roads have been a principal issue globally. From the overall roadway safety issues, a significant share has been taken by rural roads. For instance, according to data from Organization for Economic Cooperation and Development (OECD), the "average fatal crash rates per vehicle kilometer can be up to 6 times higher on 2-lane rural roads than on motorways" ("Towards Zero: Ambitious Road Safety Targets and the Safe System Approach," 2008). Even though crash rates in urban roadways are greater than in rural roads; due to higher traffic flows and the number of intersections, rural road crashes are more severe than urban ones. Poor roadway characteristics, reduced traffic enforcement, riskier roadside hazard ratings, and higher operating speeds on rural roads are the main factors that increased crash severity as compared to urban roads (Oxley et al., 2004). Thus, improving traffic safety on rural road segments is the prime most duties of safety engineers. The current study was executed in the Oromia region focusing on undivided rural two-way two-lane roadway segments. The study area is shown in Figure 1.

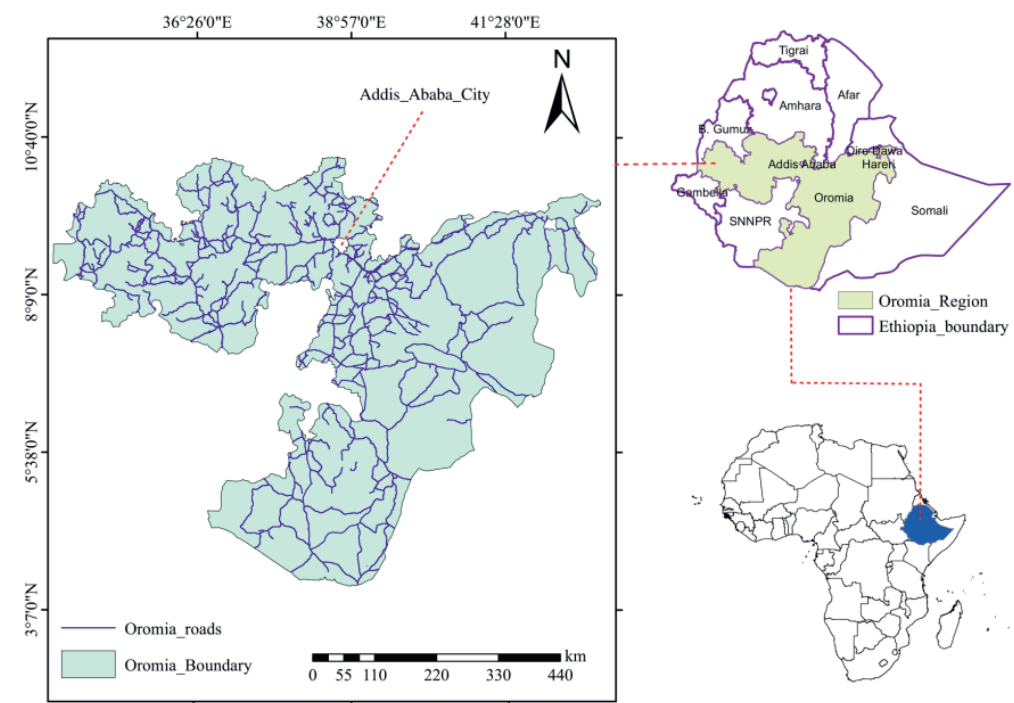

Figure 1. Map of the study area 


\subsection{Data preparation}

The procedures for data collection and preparation for analysis are described in this section. The study was carried out using primary data collection and other supplementary information. To meet the study objectives, the requested data, which are crash data and highway data (i.e. horizontal and vertical alignments, roadway cross-section, and traffic volume), were obtained.

\section{Crash data}

For the development of a fixed Oromia's crash distribution, crash data of seven consecutive years that occurred on Oromia's rural two-way two-lane roadway segments were obtained from the Oromia traffic police bureau. Furthermore, two years of crash data from 2017/18 to 2018/19 were used for comparison and validation of the developed crash distribution dataset prediction on IHSDM software. The $\mathrm{KABCO}$ scale is a severity level used in both the HSM and the IHSDM predictive method to classify various crash types. The five KABCO's severity levels are K: Fatal injury, A: Incapacitating Injury, B: Non-incapacitating Injury, C: Possible Injury, and O: No Injury crashes (PDO) ((AASHTO), 2010). In Oromia, however, there are only four crash severity levels such as; Fatal, Serious Injury, Slight Injury, and Property Damage Only (PDO). This means that while IHSDM has three severity levels (ABC), Oromia only has two (Serious Injury, Slight Injury). To make the computation easier, the percentage of C (Possible injury) for Oromia's crash data has been set to zero. This implies that the Oromia injury crashes (Serious Injury and Slight Injury) are assumed to be comparable to their equivalent IHSDMs injury crashes (Incapacitating injury, Non-incapacitating injury, and possible injury).

The nine collision types in HSM are collision with animals, collision with bicycles, collision with pedestrians, overturned, Run-Off-Road, angle collision, Head-on collision, Rear-end collision, and Sideswipe collisions. Except for bicycle collisions, all collision types are available and were obtained from the Oromia Traffic Police Bureau. The cumulative crash data from Oromia Traffic Police Bureau is presented in Table 1.

Table 1. An aggregated crash data by roadway types: From 2010/11-2016/17

\begin{tabular}{|l|l|l|l|l|}
\hline Roadway Type & Fatal & Serious Injury & Slight Injury & PDO \\
\hline One-way $(A)$ & 2334 & 1206 & 1427 & 2542 \\
\hline Two-way Undivided $(B)$ & 2724 & 1591 & 1704 & 2968 \\
\hline Divided $(C)$ & 1525 & 836 & 1147 & 2970 \\
\hline Painted with Passing Restricted $(D)$ & 907 & 604 & 534 & 1799 \\
\hline Broken-line Painted $(E)$ & 319 & 666 & 505 & 1494 \\
\hline $\begin{array}{l}\text { Total for Undivided Rural Two-way Two-lane } \\
=B+D+E\end{array}$ & 3950 & 2861 & 2743 & 6261 \\
\hline
\end{tabular}

\section{Highway data}

Highway geometric data (i.e. horizontal curve data and tangents, vertical curves and grades, and roadway cross-sections) and the general highway environments (i.e. terrain types, traffic volume, and design/posted speeds) are the fundamentals among the required data for CPM evaluation. For the sake of comparison and validation of the crash distribution dataset prediction, the as-built geometric data and the general highway environments of 'Addis Ababa' to 'Chacha' road were obtained from the Ethiopian Roads Authority (ERA). The road was selected based on data availability. Rural roads and urban/suburban roads have been differentiated based on rural and town cross-section types and populations. Accordingly, twelve undivided rural two-way two-lane segments $(71.03 \mathrm{~km})$ are identified as undivided rural two-lane roadways. IHSDM provides several options to import or create highway alignment into the tool. From these IHSDM users:

- Can manually enter roadway data using Highway Editor Tool,

- Can import highway alignment using CSV (comma separated values) format,

- Can import highway data in LandXML format from other software (i.e. MX-Road, Geopak, AutoCAD Civil 3D and others).

Using AutoCAD Civil3D software, the geometric data of the twelve undivided rural two-way twolane highway segments were imported into IHSDM. 


\section{Methods}

The focus of this research was to develop a jurisdiction-specific crash distribution dataset using crash data collected from rural two-way two-lane roads in the Oromia region. The study validated the developed crash distribution dataset prediction using HSM's default SPF and CMFs. HSM Appendix C lists five sets of default crash distribution datasets for rural two-way two-lane roads that HSM users should modify ((AASHTO), 2010). These are i) crash severity proportions, ii) collision type proportions, iii) the ratio of driveway-related crashes to overall crashes of roadway segments, iv) nighttime crash proportions of roadway segments, and v) intersection nighttime crash proportions. Except for intersections' nighttime crash proportions, all of the above-listed default crash distribution datasets were updated in this study, and a fixed crash distribution dataset for the Oromia region was finally developed.

\subsection{Crash Distribution Dataset: Development}

A seven-year crash history of the Oromia region was used to calculate the crash severity percentages, which were divided into four severity categories such as; Fatal (F), Serious Injury (SeI), Slight Injury (SI), and Property Damage Only (PDO). The computed result is presented in Figure 2(a). According to the findings, Fatal plus all Injury crashes $(\mathrm{F}+\mathrm{I})$ in Oromia are higher, accounting for $60.41 \%$ of all crashes, while the default HSM values based on crash data from the states used to develop HSM are $32.10 \%$. This demonstrates that the crashes in the Oromia region were more severe as compared to the HSM crash severity proportion. For the purposes of calculating the nighttime crash proportion, crashes that occurred at night on unlit roadways were treated as nighttime crashes for the unlighted roadways. Figure 2(b) \& Figure 2(c) depicts the computed proportion of total nighttime crashes for unlighted roadways and the severity proportion of unlighted roadway crashes based on seven years of crash history in the region.

By excluding other and unknown collision types recorded by traffic police officers, the Collision type proportion and percentages of driveway-related (Run-off-Road, Head-on collision, and Sideswipe) crashes were calculated. Based on seven years of crash data, Table 2 shows the aggregated percentage results of all collision types and the percentages of driveway-related crashes by adding the percentage of Run-off-Road, Head-on Collision, and Sideswipe Collison.

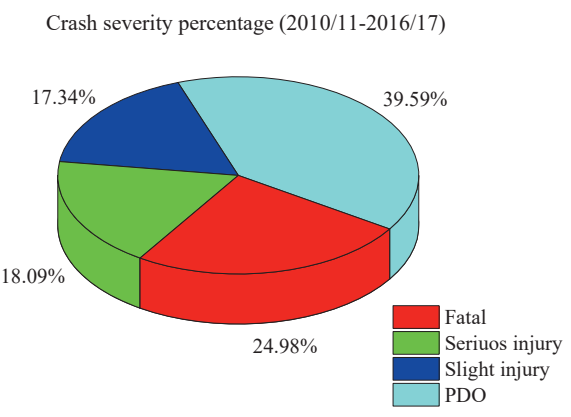

(a)

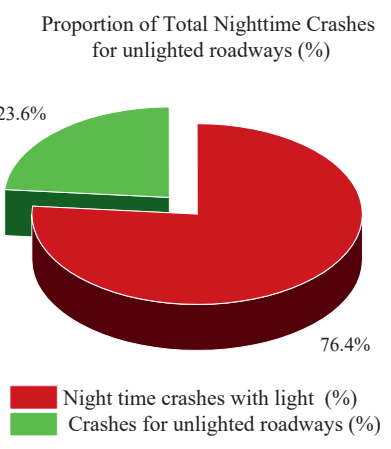

(b)

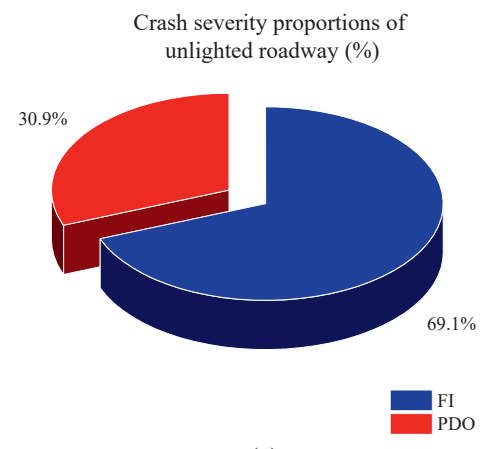

(c)

Figure 2. (a) Total crash severity percentage (b) Nighttime crash proportion for unlighted roadway (c) Unlighted roadway crash severity percentage 
Table 2. Collision type proportion and percentage of driveway related crashes

\begin{tabular}{|c|c|c|c|c|}
\hline \multirow{2}{*}{ Collision Type } & \multicolumn{2}{|l|}{ Percentage (\%) } & \multirow{2}{*}{$\begin{array}{l}\text { Percentage of Total } \\
\text { (All Severity levels } \\
\text { combined) }(\%)\end{array}$} & \multirow{2}{*}{$\begin{array}{l}\text { The proportion of total crashes } \\
\text { constituted by Run-off-the-road, } \\
\text { Head-on collision and Sideswipe (\%) }\end{array}$} \\
\hline & Fatal + Injury & PDO & & \\
\hline Collision with Animal & 5.40 & 3.10 & 4.49 & \\
\hline Collision with Pedestrians & 13.15 & 0.88 & 8.31 & \\
\hline Ran-off Road & 47.87 & 52.93 & 49.87 & 49.87 \\
\hline Overturned & 4.76 & 10.01 & 6.83 & \\
\hline Other-single Vehicle Collision & 2.72 & 2.82 & 2.76 & \\
\hline Total Single-Vehicle Crashes & 73.91 & 69.74 & 72.27 & \\
\hline Angle Collision & 5.96 & 5.08 & 5.61 & \\
\hline Head-on Collision & 5.11 & 8.72 & 6.54 & 6.54 \\
\hline Rear-end Collision & 6.67 & 8.08 & 7.22 & \\
\hline Sideswipe Collision & 4.25 & 3.88 & 4.10 & 4.10 \\
\hline Other-multiple Collision & 4.10 & 4.49 & 4.25 & \\
\hline $\begin{array}{l}\text { Total Multiple-Vehicle } \\
\text { Crashes }\end{array}$ & 26.09 & 30.26 & 27.73 & \\
\hline Total Crashes & 100.00 & 100.00 & 100.00 & 60.51 \\
\hline
\end{tabular}

\subsection{CPM evaluation}

The crash prediction module of IHSDM software was used to validate and compare the crash prediction estimates of the default HSM crash distribution datasets and the developed fixed crash distribution datasets of the Oromia region. IHSDM is a tool developed by the Federal Highway Administration (FHWA) that has been used since 1993 to assess the safety and operational effects of highway geometric designs ((FWHA), 2019). The software was first made available to the public in 2004; since then, it has been made freely available to safety practitioners and can be downloaded from the www.ihsdm.org website. The IHSDM used in this study was the latest and currently available version of the 2020 release (version of 16.0.0).

The previously (in section 3.1) computed fixed crash severity, nighttime crashes for the unlighted roadway, collision type, and total driveway-related crash proportions of the Oromia region were developed on the Administration Tool of IHSDM software as part of a calibration procedure. In addition, the AASHTO default policy was substituted by the Ethiopian Roads Authority (ERA, 2013) superelevation policy on the IHSDM Administration Tool. The comparison and validation of the developed crash distribution dataset prediction and that of the default HSM's had been carried out by running CPM of IHSDM software. The rural two-way two-lane highway data from 'Addis Ababa' to 'Chacha' (which is $71.03 \mathrm{~km}$ in length) was evaluated for the analysis period of two years (2017/18 to 2018/19) by using both the developed and default HSM's crash distribution dataset. Finally, their crash distribution predictions were compared and validated against the observed crash distribution proportions.

\section{Results and discussions}

\subsection{Crash Prediction Evaluation}

IHSDM's CPM was evaluated for all twelve rural roads imported into IHSDM software, and it was then segregated into 508 homogeneous roadway sites (roadway sites with similar characteristics) using the HSM's homogeneous roadway segregation. Out of the twelve highways evaluated, Highway-7 is presented in this section as an illustration of crash prediction module evaluation results for the developed fixed-crash distribution datasets. The exported geometric data (i.e. the horizontal alignment, vertical alignment, and cross-section) and CPM summary of Highway-7 are presented in Figure 3. For an evaluation period of two years (2017/18 to 2018/19), the IHSDM's CPM evaluation result of Highway-7 (shown in Figure 3(b)) is interpreted as follows: A total of 10.64 crash frequencies are predicted over a $6.19 \mathrm{~km}$ length. The highest crash rate, as evaluated by highway segment, is located between stations $42+531.139$ and $42+613.424$, with a predicted crash rate of $2.4743 \mathrm{crashes} / \mathrm{km} / \mathrm{yr}$, also the highest crash rate, as measured by horizontal design element, is found at simple curve $\# 5(42+500.015$ to $42+613.424)$. This means that in the assessment of Highway-7, the station from $42+500.015$ to $42+613.424$ (or simple curve \# 5) requires special treatment for crash reduction. 


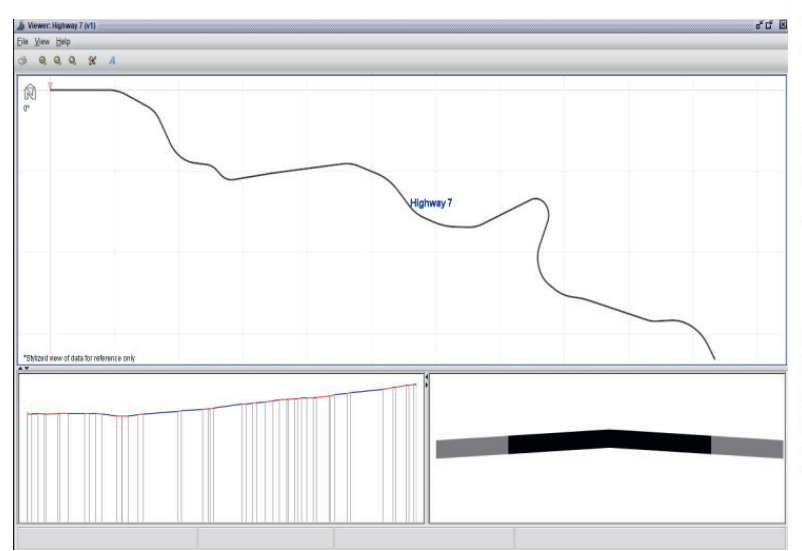

(a)

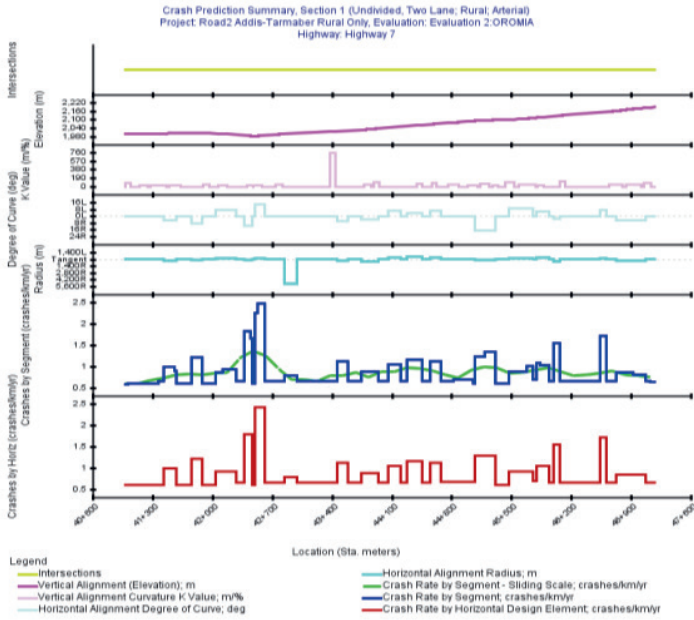

(b)

Figure 3. Highway-7 (a) geometric alignment (b) crash prediction summary

Table 3. Overall crash frequencies by collision type

\begin{tabular}{|l|l|l|l|}
\hline \multicolumn{6}{|l}{ Cumulative predicted crash frequency (All Severity) } & Oromia Configuration & HSM Configuration \\
\hline No. & Collision type & 4.54 & 12.23 \\
\hline 1 & Collision with Animal & None & 0.21 \\
\hline 2 & Collision with Bicycle & 8.41 & 0.29 \\
\hline 3 & Collision with Pedestrian & 6.62 & 1.62 \\
\hline 4 & Head-on Collision & 4.29 & 2.74 \\
\hline 5 & Other Multiple-vehicle Collision & 2.77 & 2.11 \\
\hline 6 & Other Single-vehicle Collision & 6.89 & 2.54 \\
\hline 7 & Overturned & 7.29 & 14.37 \\
\hline 8 & Rear-end Collision & 5.67 & 8.6 \\
\hline 9 & Right-Angle Collision & 50.41 & 52.68 \\
\hline 10 & Run-off-Road & 4.16 & 3.74 \\
\hline 11 & Sideswipe & 28.03 & 31.04 \\
\hline 12 & Total Multiple Vehicle Crashes & 73.07 & 70.09 \\
\hline 13 & Total Single-Vehicle Crashes & 101.08 & 101.13 \\
\hline 14 & Total Highway Segment Crashes & & \\
\hline
\end{tabular}

The CPM of IHSDM was evaluated for each of the twelve rural roads independently, using the same evaluation period and procedure as Highway-7. The developed fixed-crash distribution dataset, as well as the default HSM configuration, were used to predict crash proportions, and the final summated crash frequency results for all highways are exhibited in Table 3 and Table 4, in their respective crash distribution datasets. Table 3 summarizes the predicted crash frequencies of all highways based on collision type, whereas Table 4 shows the predicted crash frequencies of individual highways based on severity type. Run-off-Road collision types were more common in both states, such as Oromia and states used to develop HSM crash configuration.

\subsection{Comparison and Validation for the estimates of Crash Distribution Datasets}

The observed crash data from 2017/18 to 2018/19 have been documented on the Administration tool of IHSDM to compare and validate the developed crash distribution dataset prediction. Crash severity percentages and collision type proportions of the observed data are illustrated in Figure 4. Figure 5 compares the developed, HSM, and observed crash distribution datasets in terms of crash severity percentage and collision type proportion. Based on this comparison, the developed and observed crash severity percentages are much closer, whereas the HSM crash severity percentage has significantly deviated from the observed. 


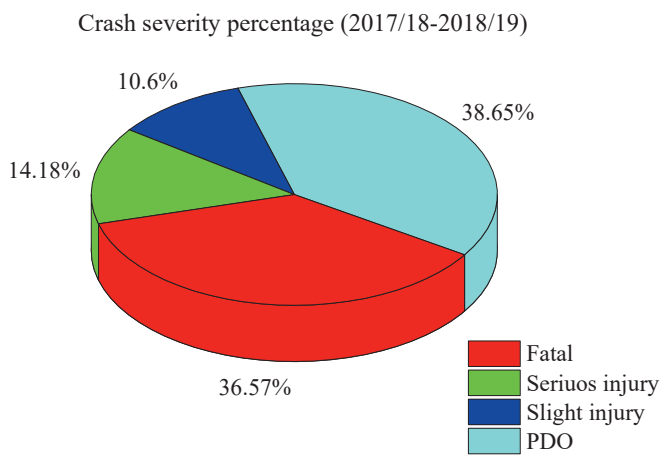

(a)

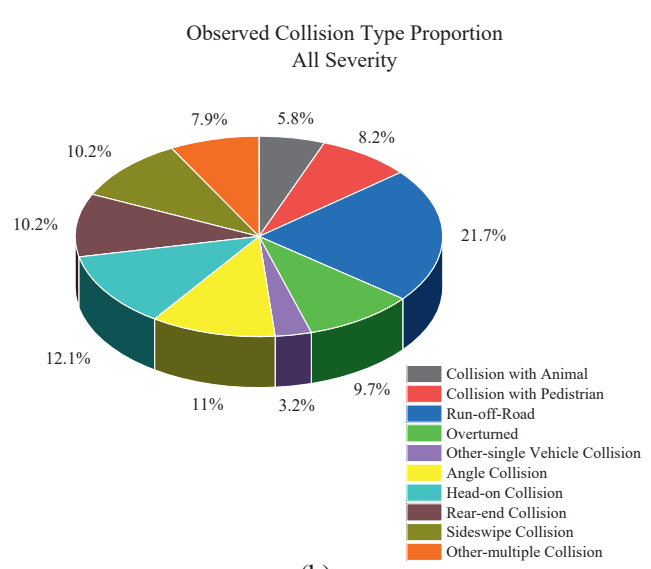

(b)

Figure 4. The (a) observed crash severity proportions (b) observed collision type proportion for all severity types

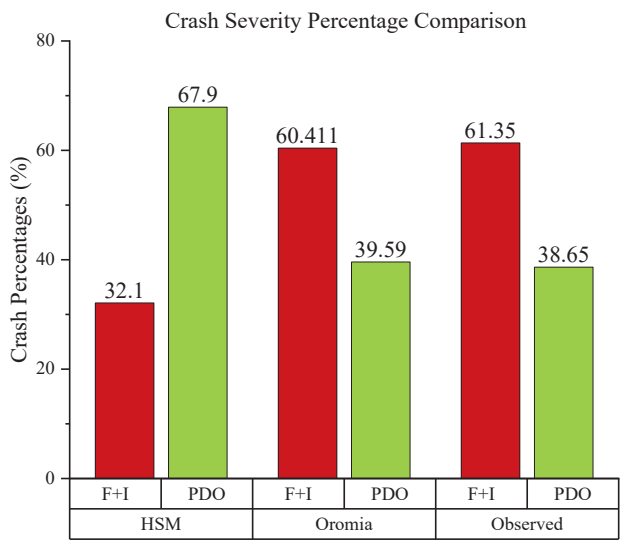

(a)

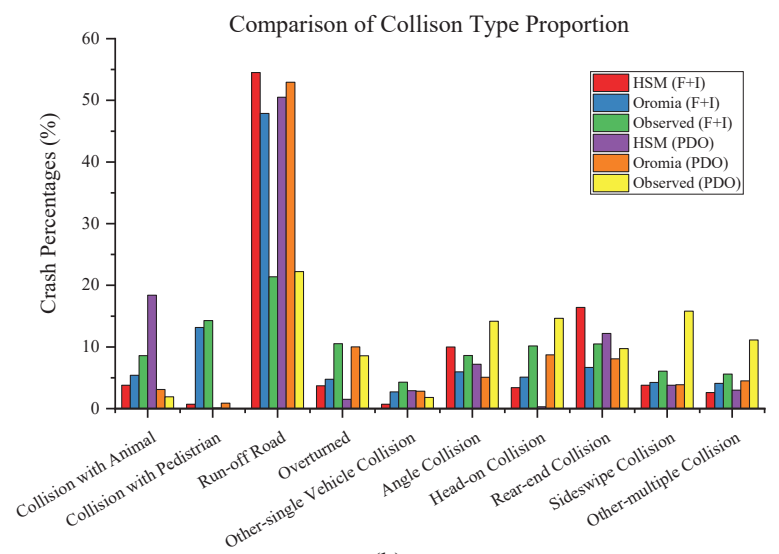

(b)

Figure 5. The comparison of (a) crash severity percentage (b) collision type proportion

Goodness-of-fit statistics, such as Mean Absolute Difference, MAD and Symmetric Mean Absolute Percentage Error, SMAPE, as given in Equation (2) and Equation (3) were used to compare and validate crash distribution predictions from both the default HSM and the developed Fixed-Oromia's crash distribution datasets. The closer the values of $M A D$ and $S M A P E$ are to zero, the more accurately or close to the observed data the crash distribution configuration predicts. The closer the values of $M A D$ and $S M A P E$ are to zero, the more accurate is the crash distribution dataset prediction closer to the observed data. For both crash severity proportions and collision type distributions, the prediction accuracy of the default HSM configuration and that of the developed has been quantified. The results are exhibited and discussed as follows.

$$
\begin{aligned}
& M A D=\frac{1}{n} \sum_{i=1}^{n}|Y-\bar{Y}| . \\
& S M A P E=\frac{1}{n} \sum_{i=1}^{n} \frac{|Y-\bar{Y}|}{Y+\bar{Y}} * 100 .
\end{aligned}
$$

Where $Y$ is the observed value and $\bar{Y}$ is the predicted value.

\section{Crash severity percentage}

Table 4 summarizes and presents the crash severity percentages of all highways predicted by the IHSDM crash prediction model for the developed and default HSM configurations. In addition, for comparison, the observed crash severity percentage data is included in the table. Whenever the observed crashes for a specific site are known, a calibration factor, $C_{x}$ in Equation (4), can be computed. The 
calibration factor for fatal and injury crashes $(\mathrm{F}+\mathrm{I})$ calculated using Equation (4) was 1.018 for the developed configuration and 1.915 for that of the HSM. Both configurations underpredict Fatal and Injury crashes $(\mathrm{F}+\mathrm{I})$, with the Oromia configuration underpredicting in $1.8 \%$ and the HSM configuration underpredicting in $91.5 \%$. These calibration factor computations show that the developed configuration outperforms the HSM in terms of predicting crash severity percentage.

$C_{x}=\frac{\sum N_{\text {Observed }}}{\sum N_{\text {Predicted }}}$.

Where $C_{x}$ is a calibration factor, $N_{\text {Observed }}$ is the observed crash proportion and $N_{\text {Predicted }}$ is the predicted crash proportion.

Table 4. Crash severity proportions summarized by highway number

\begin{tabular}{|c|c|c|c|c|c|}
\hline Highway No. & Length (KM) & Severity type & Observed & Oromia Configuration & HSM Configuration \\
\hline \multirow{2}{*}{1} & \multirow{2}{*}{3.7} & All & 5.09 & 5.08 & 5.08 \\
\hline & & FI & 3.12 & 3.07 & 1.63 \\
\hline \multirow{2}{*}{2} & \multirow{2}{*}{2.275} & All & 4.1 & 4.09 & 4.09 \\
\hline & & FI & 2.52 & 2.47 & 1.31 \\
\hline \multirow{2}{*}{3} & \multirow{2}{*}{0.95} & All & 1.17 & 1.16 & 1.16 \\
\hline & & FI & 0.72 & 0.7 & 0.37 \\
\hline \multirow{2}{*}{4} & \multirow{2}{*}{6.06} & All & 7.84 & 7.82 & 7.83 \\
\hline & & FI & 4.81 & 4.72 & 2.51 \\
\hline \multirow{2}{*}{5} & \multirow{2}{*}{2.35} & All & 3.57 & 3.56 & 3.56 \\
\hline & & FI & 2.19 & 2.15 & 1.14 \\
\hline \multirow{2}{*}{6} & \multirow{2}{*}{10.6} & All & 15.78 & 15.74 & 15.75 \\
\hline & & FI & 9.68 & 9.51 & 5.06 \\
\hline \multirow{2}{*}{7} & \multirow{2}{*}{6.195} & All & 10.66 & 10.64 & 10.64 \\
\hline & & FI & 6.54 & 6.43 & 3.42 \\
\hline \multirow{2}{*}{8} & \multirow{2}{*}{8.35} & All & 10.7 & 10.67 & 10.68 \\
\hline & & FI & 6.56 & 6.45 & 3.43 \\
\hline \multirow{2}{*}{9} & \multirow{2}{*}{4.25} & All & 5.45 & 5.44 & 5.44 \\
\hline & & FI & 3.34 & 3.29 & 1.75 \\
\hline \multirow{2}{*}{10} & \multirow{2}{*}{10.65} & All & 15.17 & 15.14 & 15.15 \\
\hline & & FI & 9.31 & 9.15 & 4.86 \\
\hline \multirow{2}{*}{11} & \multirow{2}{*}{6.3} & All & 8.88 & 8.86 & 8.87 \\
\hline & & FI & 5.45 & 5.35 & 2.85 \\
\hline \multirow{2}{*}{12} & \multirow{2}{*}{9.35} & All & 12.9 & 12.88 & 12.88 \\
\hline & & FI & 7.91 & 7.78 & 4.13 \\
\hline
\end{tabular}

Aside from calibration factor computations, goodness-of-fit statistics were used to compare and validate the developed crash distribution estimates in terms of severity type. As detailed in Table 5, the resulted MAD and SMAPE goodness-of-fit statistics show that the developed Oromia's crash distribution dataset predicts both FI and PDO crash severity proportions more accurately than the HSM. For FI crash estimates, the developed configuration yielded a MAD of 0.09 , which is much closer to zero than the HSM configuration, which yielded a MAD of 2.4742. Similarly, the developed configuration had a SMAPE of $0.9199 \%$ for FI prediction, while the HSM configuration had a higher SMAPE of $31.4488 \%$, which is too far from zero. The efficacy of the developed crash distribution dataset to predict severity percentages in the study area was proven by the goodness-of-fit statistics employed in this study.

Table 5. Performance measures of predicting crashes by severity proportions

\begin{tabular}{|l|l|l|l|}
\hline Severity type & A measure of prediction accuracy & Oromia Configuration & HSM Configuration \\
\hline FI & MAD & 0.0900 & 2.4742 \\
\hline & SMAPE (\%) & 0.9199 & 31.4488 \\
\hline PDO & MAD & 0.0708 & 2.4592 \\
\hline & SMAPE (\%) & 1.0785 & 27.3722 \\
\hline
\end{tabular}




\section{Collision type distribution}

Exclusive of Other Single-vehicle Collisions and Other Multiple-vehicle Collisions, Collision with Animal, Collision with Bicycle, Collision with Pedestrian, Head-on Collision, Overturned, Rear-end Collision, Angle Collision, Run-off-Road, and Sideswipe are the nine collision types in HSM and IHSDM. Eight of the nine collision types have been recorded and classified in the Oromia crash recording system, except for the Collision with Bicycle, which has not been specifically recorded. Thus, Collision with Bicycle was omitted for model calibration, and in addition to the eight collision types, Other Singlevehicle Collisions and Other Multiple-vehicle Collisions were added, totaling ten collision types evaluated. The crash prediction results of the twelve rural roads were aggregated in terms of their respective collision type distribution, and the final summated values for both crash distribution datasets were statistically assessed for goodness-of-fit. Table 6 and Table 7 show the detailed goodness-of-fit statistics computed for the collision type prediction accuracy of both configurations.

Table 6. Performance measures of collision type prediction categorized in FI severity

\begin{tabular}{|c|c|c|c|c|c|}
\hline \multirow{3}{*}{ No. } & \multirow{3}{*}{ Collision type } & \multicolumn{4}{|l|}{ FI } \\
\hline & & \multicolumn{2}{|c|}{ Oromia Configuration } & \multicolumn{2}{|c|}{ HSM Configuration } \\
\hline & & MAD & SMAPE & MAD & SMAPE \\
\hline 1 & Collision with Animal & 0.1692 & 0.2318 & 0.3425 & 0.6331 \\
\hline 2 & Collision with Pedestrian & 0.0733 & 0.0522 & 0.7225 & 0.9548 \\
\hline 3 & Head-on Collision & 0.2650 & 0.3312 & 0.4333 & 0.7031 \\
\hline 4 & Other Multiple-vehicle Collision & 0.0800 & 0.1574 & 0.2192 & 0.6131 \\
\hline 5 & Other Single-vehicle Collision & 0.0825 & 0.2253 & 0.2025 & 0.8564 \\
\hline 6 & Overturned & 0.2633 & 0.3513 & 0.3883 & 0.6369 \\
\hline 7 & Rear-end Collision & 0.2017 & 0.2243 & 0.0958 & 0.0947 \\
\hline 8 & Right-Angle Collision & 0.1425 & 0.1912 & 0.1717 & 0.2352 \\
\hline 9 & Run-off-Road & 1.3283 & 0.3756 & 0.3658 & 0.1417 \\
\hline 10 & Sideswipe & 0.0983 & 0.1829 & 0.2108 & 0.5136 \\
\hline
\end{tabular}

Table 7. Performance measures of collision type prediction categorized in All severity

\begin{tabular}{|l|l|l|l|l|l|}
\hline \multirow{2}{*}{ No. } & \multirow{2}{*}{ Collision type } & \multicolumn{2}{l|}{ All Severity } & \multicolumn{2}{l|}{ HSM Configuration } \\
\cline { 3 - 7 } & & \multicolumn{2}{l|}{ Oromia Configuration } & \multicolumn{2}{l|}{ MAD } \\
\cline { 3 - 7 } & & MAD & SMAPE & \multicolumn{2}{l|}{ MAPE } \\
\hline 1 & Collision with Animal & 0.1083 & 0.1294 & 0.5325 & 0.3515 \\
\hline 2 & Collision with Pedestrian & 0.0050 & 0.0035 & 0.6717 & 0.9388 \\
\hline 3 & Head-on Collision & 0.4642 & 0.2943 & 0.8808 & 0.7629 \\
\hline 4 & Other Multiple-vehicle Collision & 0.3117 & 0.3023 & 0.4408 & 0.4903 \\
\hline 5 & Other Single-vehicle Collision & 0.0417 & 0.0870 & 0.0967 & 0.2252 \\
\hline 6 & Overturned & 0.2133 & 0.1600 & 0.5292 & 0.5380 \\
\hline 7 & Rear-end Collision & 0.2500 & 0.1731 & 0.3400 & 0.1658 \\
\hline 8 & Right-Angle Collision & 0.4533 & 0.3229 & 0.2092 & 0.1279 \\
\hline 9 & Run off-Road & 2.3633 & 0.3916 & 2.5525 & 0.4098 \\
\hline 10 & Sideswipe & 0.5150 & 0.4247 & 0.5500 & 0.4714 \\
\hline
\end{tabular}

From the ten collision type distribution datasets developed for Fatal and Injury (FI) crash predictions, the HSM's crash distribution dataset predicted Run-off-Road and Rear-end Collision relatively well, while the rest was predicted more accurately by the developed crash distribution datasets closer to the observed data. Except for Collisions with Pedestrians and Sideswipe Collisions, the developed crash distribution dataset prediction consistently outperforms the HSM in the overall crash severity type evaluation. The developed crash distribution dataset for predicting crash frequencies in terms of both severity and collision type proportions for the study's jurisdiction has been validated, and the results confirm its effectiveness. As a result, the systematic approach used in this study to develop a jurisdiction crash distribution dataset is capable of fixing the error caused by the use of the default HSM's dataset. 


\section{Conclusions}

Examining the safety performance of roadways, such as CPM evaluation, for specific treatments is critical in improving highway safety. The FHWA developed the predictive HSM to quantify the expected average crash frequency that may result from a specific highway feature; however, the default HSM may be unreliable for use by local jurisdictions other than those used to develop HSM. The predictive HSM method predicts traffic crashes based on the proportions of severity and collision type. A jurisdictionspecific crash distribution dataset must be developed and used in the evaluation to incorporate crash severity and collision type proportions in the crash prediction model. This study developed a fixed Oromia's crash distribution dataset and evaluated its effectiveness by replacing the default HSM values on the Administration tool of IHSDM software. The crash dataset was developed using crash data from rural two-lane road segments in the Oromia region collected over seven years (from 2010/11 to 2016/17). The crash distribution dataset development included both estimates of the crash severity percentages and the collision type proportions.

The study result shows that the developed crash distribution dataset, when compared to the default HSM dataset, predicts crash severity proportions much closer to the observed values. For Fatal and Injury crashes $(\mathrm{F}+\mathrm{I})$, the calibration factors for the developed and HSM configurations are 1.018 and 1.915, respectively. Both configurations underpredict fatal and injury crashes, with the Oromia configuration underpredicting in $1.8 \%$ and the HSM configuration underpredicting in $91.5 \%$. According to the calibration factors obtained above, crashes in the Oromia region are severe as compared to the states used to develop the HSM configuration. Therefore, at this point, the authors imply that transportation authorities, traffic police bureaus, safety practitioners, and other concerned parties still have a lot of work to do to reduce the severity of crashes across the region. In addition to the calibration factor results, the goodness-of-fit statistics used in this study confirmed the accuracy of the developed crash distribution dataset in predicting crash severity percentages in the study area. Furthermore, the study results in the collision type predictions part show that the developed crash distribution dataset predicts much better than the default HSM, but not in all collision type proportions. The default HSM configuration, for example, predicts Right-Angle and Sideswipe Collision proportions more precisely from the ten collision type proportions developed. Based on the availability of complete crash data (i.e. crash location), the result points to the need for developing collision type proportion as a function rather than a fixed configuration for a better result.

By adopting and following the procedures in the Highway Safety Manual, the target of highway safety improvement becomes more achievable for the predictive safety analysis based on the performance method. The predictive Highway Safety Manual and IHSDM software need to be adopted in Oromia for a better road safety study. However, to obtain accurate and locally feasible crash estimates in Oromia, the default HSM crash distribution dataset must have to be substituted by the developed crash configuration in this study. As demonstrated in this study, unsubstituted default HSM configuration with the locally developed one may result in inaccurate and unrealistic crash distribution estimates and emasculate its liability.

In general, the current study assures practitioners that crash distribution datasets vary from jurisdiction to jurisdiction due to differences in geographic conditions, crash reporting systems, road users characteristics (i.e., pedestrians, animals, and drivers behavior), vehicle characteristics, climate conditions and other undetected factors. A statement is made that, in order to better suit local conditions, safety practitioners should develop the jurisdiction's crash distribution dataset using the methodologies demonstrated and validated as true in this study, rather than using the default HSM crash distribution dataset. Crash prediction models that are based on an accurate jurisdiction's crash distribution (i.e., by severity level and collision type) investigate the relationships between roadway geometric features and their associated crash types, thus, assisting policymakers, road safety engineers, and transport agencies in making effective improvements on the severer crashes.

\section{References}

1. (AASHTO), A. A. of S. H. and T. O. (2010) Highway Safety Manual (First). American Association of State Highway and Transportation Officials.

2. (FWHA), F. H. A. (2019) Interactive Highway Safety Design Model (IHSDM): Overview. U.S. Department of Transportation, Federal Highway Administration. Available: https://highways.dot.gov/research/safety/interactive-highway-safety-design-model/interactivehighway-safety-design-model-ihsdm-overview 
3. Abdel-Aty, M. A., Lee, C., Park, J., Wang, J.-H., Abuzwidah, M. \& Al-Arif, S. (2014) Validation and application of highway safety manual (Part D) in Florida (No. BDK78-977-14; Issue May). Florida Department of Transportation.

Available: http://www.cmfclearinghouse.org/study_detail.cfm?stid=433

4. Abdel-Aty, M. \& Keller, J. (2005) Exploring the overall and specific crash severity levels at signalized intersections. Accident Analysis and Prevention, 37(3), 417-425. DOI:10.1016/j.aap.2004.11.002

5. Ahmed, M. \& Chalise, R. (2018) Calibration of the Highway Safety Manual's Safety Performance Functions for Rural Two-Lane Highways with Regional Considerations for the Rocky Mountains and Plain Regions (MPC-18-344; MPC-18-344).

Available: http://www.ugpti.org/resources/reports/details.php?id=901

6. Brimley, B., Saito, M. \& Schultz, G. (2012) Calibration of highway safety manual safety performance function. Transportation Research Record, 2279, 82-89. DOI:10.3141/2279-10

7. Burgess, M. (2005) Contrasting Rural and Urban Fatal Crashes 1994-2003. In U.S. Dept. of Transportation, National Highway Traffic Safety Administration (Issue DOT HS 809 896). Available: http://purl.access.gpo.gov/GPO/LPS97980

8. Chandler, B. \& Anderson, R. (2010) Implementing the High Risk Rural Roads Program. In U.S. Federal Highway Administration (FHWA) (Issue FHWA-SA-10-012). Available: http://safety.fhwa.dot.gov/local_rural/training/fhwasa10012/fhwasa10012.pdf

9. Dominguez-Lira, C. A., Castro, M., Pardillo-Mayora, J. M. \& Gascon-Varon, C. (2010) Adaptation and Calibration of IHSDM for Highway Projects Safety Evaluation in Spain. Proceedings of the 4th International Symposium on Highway Geometric Design, 12. Available: https://trid.trb.org/view/1098951

10. Fitzpatrick, K., Schneider IV, W. \& Carvell, J. (2006) Using the rural two-lane highway draft prototype chapter. Transportation Research Record, 1950, 44-54. DOI:10.3141/1950-06

11. Haleem, K. \& Abdel-Aty, M. (2010) Examining traffic crash injury severity at unsignalized intersections. Journal of Safety Research, 41(4), 347-357. DOI:10.1016/j.jsr.2010.04.006

12. Huang, H., Chin, H. C. \& Haque, M. M. (2008) Severity of driver injury and vehicle damage in traffic crashes at intersections: A Bayesian hierarchical analysis. Accident Analysis and Prevention, 40(1), 45-54. DOI:10.1016/j.aap.2007.04.002

13. Jonsson, T., Lyon, C., Ivan, J. N., Washington, S. P., Van Schalkwyk, I. \& Lord, D. (2009). Differences in the performance of safety performance functions estimated for total crash count and for crash count by crash type. Transportation Research Record, 2102, 115-123. DOI:10.3141/2102-15

14. Koorey, G. (2010) Calibration of Highway Crash Prediction Models for other Countries - a Case Study with IHSDM. 4th International Symposium on Highway Geometric Design, 16. Available: https://trid.trb.org/view/1098932

15. Kweon, Y. J., Lim, I. K., Turpin, T. L. \& Read, S. W. (2014) Guidance on customization of highway safety manual for Virginia: Development and application. Transportation Research Record, 2435, 27-36. DOI:10.3141/2435-04

16. Marchionna, A., Perco, P. \& Falconetti, N. (2012) Evaluation of the Applicability of IHSDM Crash Prediction Module on Italian Two-Lane Rural Roads. Procedia - Social and Behavioral Sciences, 53, 933-942. DOI:10.1016/j.sbspro.2012.09.942

17. Martinelli, F., La Torre, F. \& Vadi, P. (2009) Calibration of the highway safety manual's accident prediction model for italian secondary road network. Transportation Research Record, 2103, 1-9. DOI:10.3141/2103-01

18. Mehta, G. \& Lou, Y. (2013) Calibration and development of safety performance functions for alabama. Transportation Research Record, 2398, 75-82. DOI:10.3141/2398-09

19. Oxley, J., Corben, B., Koppel, S., Fildes, B., Jacques, N., Symmons, M. \& Johnston, I. (2004) CostEffective Infrastructure Measures on Rural Roads (Report No. 217; Issue Report No. 217). Monash University Accident Research Centre. Available: https://www.monash.edu/muarc/archive/ourpublications/reports/muarc217

20. Park, J. \& Abdel-Aty, M. (2017) Safety performance of combinations of traffic and roadway crosssectional design elements at straight and curved segments. Journal of Transportation Engineering, 143(6), 1-9. DOI:10.1061/JTEPBS.0000033

21. Persaud, B., Lord, D. \& Palmisano, J. (2002) Calibration and transferability of accident prediction models for urban intersections. Transportation Research Record, 1784, 57-64. DOI:10.3141/1784-08

22. Persaud, B. N., Retting, R. A. \& Lyon, C. A. (2004) Crash reduction following installation of centerline rumble strips on rural two-lane roads. Accident Analysis and Prevention, 36, 1073-1079. DOI:10.1016/j.aap.2004.03.002 
23. Persaud, B., Saleem, T., Faisal, S., Lyon, C., Chen, Y. \& Sabbaghi, A. (2012) Adoption of Highway Safety Manual Predictive Methodologies Canadian Highways. "Implementation of Predictive Methods in the Procedures and Processes of Transportation Agencies" Session of the 2012 Conference of the Transportation Association of Canada Fredericton, 1-30. Available: http://conf.tac-atc.ca/english/annualconference/tac2012/docs/session15/persaud.pdf

24. Qin, X., Chen, Z., Cutler, C. \& Vachal, K. (2013) Evaluating Local and Tribal Rural Road Design with the Interactive Highway Safety Design Model ( IHSDM) (DP-264; Issue September). North Dakota State University, Upper Great Plains Transportation Institute.

25. Qin, X., Chen, Z. \& Shaon, R. R. (2019) Developing jurisdiction-specific SPFs and crash severity portion functions for rural two-lane, two-way intersections. Journal of Transportation Safety and Security, 11(6), 629-641. DOI:10.1080/19439962.2018.1458052

26. Qin, X., Shaon, M. R. R. \& Chen, Z. (2016) Developing analytical procedures for calibrating the Highway Safety Manual predictive methods. Transportation Research Record, 2583, 91-98. DOI:10.3141/2583-12

27. Sacchi, E., Persaud, B. \& Bassani, M. (2012) Assessing International Transferability of Highway Safety Manual Crash Prediction Algorithm and Its Components. Transportation Research Record: Journal of the Transportation Research Board. DOI:10.3141/2279-11

28. Sawalha, Z. \& Sayed, T. (2006) Transferability of accident prediction models. Safety Science, 44(3), 209-219. DOI:10.1016/j.ssci.2005.09.001

29. Sun, C., Edara, P., Brown, H., Zhu, Z. \& Rahmani, R. (2014) Calibration of Highway Safety Manual Work Zone Crash Modification Factors. In Smart Work Zone Deployment Initiative (InTrans Project 06-277; Iowa DOT TPF-5(081); Vol. 5, Issue June). InTrans Project Reports. Available: https://lib.dr.iastate.edu/intrans_reports/88

30. Sun, X., Li, Y., Magri, D. \& Shirazi, H. H. (2006) Application of highway safety manual draft chapter: Louisiana experience. Transportation Research Record, 1950, 55-64. DOI:10.3141/1950-07

31. Tang, E., Peach, K., DeFisher, J. \& Eccles, K. (2018) Transportation Safety Planning and the Zero Deaths Vision: A Guide for Metropolitan Planning Organizations and Local Communities (Issue FHWA-SA-18-024). Federal Highway Administration (FHWA). Available: https://rosap.ntl.bts.gov/view/dot/43671

32. Tarko, A. P., Romero, M., Hall, T. \& Sultana, A. (2018) Updating the Crash Modification Factors and Calibrating the IHSDM for Indiana (Publication No. FHWA/IN/JTRP-2018/03; Publication No. FHWA/IN/JTRP-2018/03). Joint Transportation Research Program Publication No. FHWA/IN/JTRP-2018/03. DOI:10.5703/1288284316646

33. Tola, A. M. \& Gebissa, A. (2019) Assessment on the Impacts of Road Geometry and Route Selection on Road Safety: A Case of Mettu-Gore Road, Ethiopia. American Journal of Civil Engineering and Architecture, 7(1), 13-19. DOI:10.12691/ajcea-7-1-2

34. Towards zero: Ambitious road safety targets and the safe system approach. (2008) In: Towards Zero: Ambitious Road Safety Targets and the Safe System Approach (Vol. 9789282101). Organization for Economic Cooperation and Development (OECD). DOI:10.1787/9789282101964-en

35. Xie, F., Gladhill, K., Dixon, K. \& Monsere, C. (2011) Calibration of highway safety manual predictive models for oregon state highways. Transportation Research Record, 2241, 19-28. DOI:10.3141/2241-03

36. Zegeer, C. V., Reinfurt, D. W., Hummer, J., Herf, L. \& Hunter, W. (1988) Safety effects of crosssection design for two-lane roads. Transportation Research Record, 1195, 20-32. Available: https://trid.trb.org/view/302144 